CRYSTALLOGRAPHIC COMMUNICATIONS

ISSN 2056-9890

Received 13 March 2019

Accepted 25 March 2019

Edited by C. Rizzoli, Universita degli Studi di Parma, Italy

Keywords: crystal structure; copper complex; imidazolidine ligand.

CCDC reference: 1905520

Supporting information: this article has supporting information at journals.iucr.org/e

\section{Chlorido(2,2'-\{[2-(1-methyl-1H-imidazol-2-yl- $\left.\kappa N^{3}\right)$ - imidazolidine-1,3-diyl- $\kappa$ N]bis(methylene) \}bis(1- methyl-1H-imidazole- $\left.\kappa N^{3}\right)$ )copper(II) perchlorate}

\author{
Diego da Silva Padilha and Marciela Scarpellini*
}

Instituto de Química, Universidade Federal do Rio de Janeiro, Av. Athos da, Silveira Ramos, 149, BI. A, Lab. 628a. CEP 21941-909, Rio de Janeiro, RJ, Brazil. *Correspondence e-mail: marciela@iq.ufrj.br

In the crystal structure of the title complex, $\left[\mathrm{CuCl}\left(\mathrm{C}_{17} \mathrm{H}_{24} \mathrm{~N}_{8}\right)\right] \mathrm{ClO}_{4}$, the copper(II) metal exhibits an $\mathrm{N}_{4} \mathrm{Cl}$ pentacoordinate environment in a distorted square-pyramidal geometry. Coordination to the metal centre occurs through the three 1-methylimidazole $\mathrm{N}$ atoms from the pendant groups, one amine $\mathrm{N}$ atom from the imidazolidine moiety and one chlorido anion. Intermolecular interactions take place at two of the 1-methyl-imidazole rings in the form of parallel-displaced $\pi-\pi$ stacking interactions forming chains parallel to the $a$ axis. Three $\mathrm{O}$ atoms of the perchlorate anion are rotationally disordered between two orientations with occupancies of 0.5 .

\section{Chemical context}

Copper ions play a key role in many natural processes, as they are found in the active site of enzymes involved in electron and $\mathrm{O}_{2}$ transfers, oxidation and reduction, being a target for the obtaining of biomimetic or bioinspired compounds (Stephanos \& Addison, 2014). As a result of the redox characteristics of the copper ion, the versatility of ligands to which it coordinates, and the geometries it is capable of forming, copper complexes have attracted attention as catalysts for different transformations, mainly involving the activation and reduction of oxygen (Elwell et al., 2017). For the hydrogen evolution reaction (HER), the obtaining of homogeneous copper catalysts is limited by the dissociation of copper(II) because of the more negative potentials required for the reduction of protons (Zhang et al., 2014; Du et al., 2016). However, different copper complexes have been obtained and evaluated as catalysts for HER, showing promising results (Zhang et al., 2016; Haddad et al., 2017; Khusnutdinova et al., 2018). The use of bioinspired tripodal tetradentate ligands in the construction of metal complexes catalysts can provide a unique feature, the presence of cis-labile sites for substrate coordination that may be a requisite for its catalytic activity, facilitating electron/atom transfer processes.

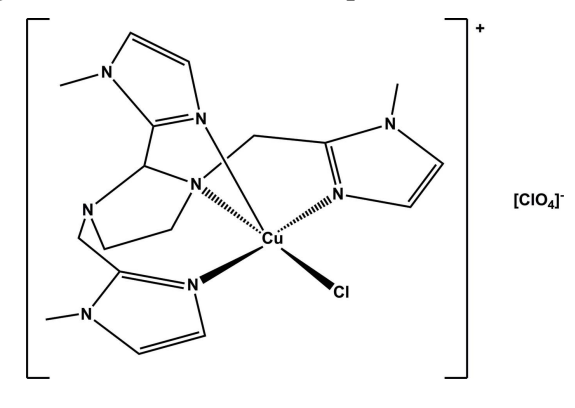


Herein, we report the molecular and crystal structure of a novel mononuclear copper(II) complex bearing an imidazolidine tetradentate ligand, namely chlorido $\left(2,2^{\prime}-\{[2-(1-m e t h y l-\right.$ $1 H$-imidazol-2-yl- $\kappa N^{3}$ )imidazolidine-1,3-diyl- $\left.\kappa N\right]$ bis(methylene)\}bis(1-methyl- $1 H$-imidazole- $\left.\kappa N^{3}\right) \operatorname{copper}(\mathrm{II})$ perchlorate, $[\mathrm{Cu}(L) \mathrm{Cl}] \mathrm{ClO}_{4}$. Similar complexes obtained with pentadentate ligands derived from the imidazolidine ring opening were previously reported (Cisnetti et al., 2007; Garcia et al., 2015), but to the best of our knowledge, this is the first example of a copper complex bearing an imidazolidine ligand with three 1-methyl-imidazole side arms.

\section{Structural commentary}

The title complex crystallizes in the monoclinic system, space group $P 21 / n$. The asymmetric unit comprises one complex cation and one disordered perchlorate anion (Fig. 1). The copper(II) ion has an $\mathrm{N}_{4} \mathrm{Cl}$ pentacoordinated environment formed by one ligand molecule and one chlorido ion. Coordination of the ligand to the metal centre occurs through the three 1-methyl-imidazole nitrogen atoms $\left(\mathrm{N}_{\mathrm{Me} \text {-im }}\right)$ and one of the tertiary amine nitrogen atoms from the imidazolidine moiety $\left(\mathrm{N}_{\mathrm{am}}\right)$. A distorted square-pyramidal geometry is observed $(\tau=0.39)$, with the basal plane composed of the chlorido ion, the amine nitrogen and the two equivalent 1-methyl-imidazole nitrogen atoms N11/N21. The third 1-methyl-imidazole nitrogen N31 occupies the apical position. Distortion of the geometry is evidenced by the bond angles in the coordination sphere, ranging from $77.43(11)$ to $113.64(12)^{\circ}$ and $147.65(12)$ to $171.21(8)^{\circ}$ for the cis and trans angles, respectively. This highly distorted square-pyramidal geometry may arise from the formation of a seven-membered chelate ring $(\mathrm{Cu} 1 / \mathrm{N} 1 / \mathrm{C} 5 / \mathrm{N} 4 / \mathrm{C} 7 / \mathrm{C} 32 / \mathrm{N} 31)$ that is less tensioned than the four or five-membered rings, allowing a more flexible arrangement. As a consequence of the geometry

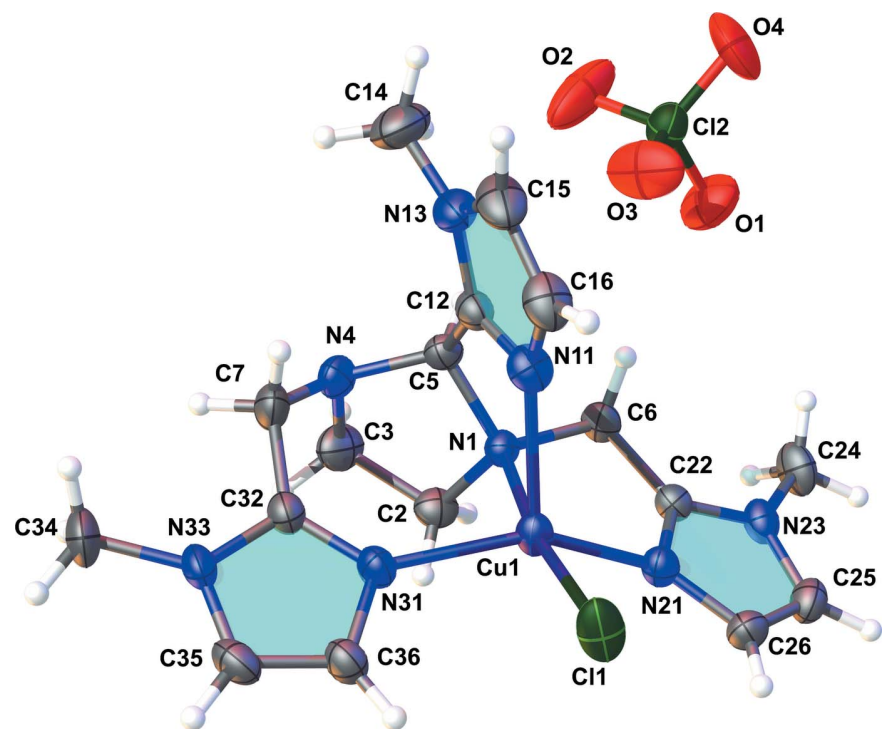

Figure 1

The structure of the title compound with displacement ellipsoids drawn at the $50 \%$ probability level. Only one component of the disordered oxygen atoms of the perchlorate anion is shown. distortion, the copper(II) ion lies 0.2565 (13) $\AA$ above the Cl1/ N21/N1/N11 basal plane towards the apical position. Squarepyramidal copper complexes exhibiting a smaller geometry distortion tends to show a slighter displacement of the metal centre from the basal plane. In the similar $[\mathrm{Cu}(\mathrm{bpqa}) \mathrm{Cl}]^{+}(\tau=$ $0.16)$ and $[\mathrm{Cu}(\mathrm{tmqa}) \mathrm{Cl}]^{+}(\tau=0.06)$ complexes $[\mathrm{bpqa}=1-$ (pyridin-2-yl)- $N$-(pyridin-2-ylmethyl)- $N$-(quinolin-2-ylmethyl)methanamine; tmqa = tris(quinolin-2-ylmethyl)amine; Wei et al., 1994], the copper(II) ion is 0.189 (2) and 0.045 (3) above the basal plane, respectively. For the complexes $[\mathrm{Cu}(L)(\mathrm{ONO})]^{+}(\tau=0.27 ; \quad L=$ bis(2-methylimidazol-2yl)methyl][2-(pyridyl-2-yl)ethyl]amine; Scarpellini et al., $2004 a)$ and $\left[\mathrm{Cu}\left(\right.\right.$ Hhis-im $\left.{ }_{2}\right) \mathrm{Cl}^{+}\left\{\tau=0.31\right.$; Hhis-im ${ }_{2}=2-(1 \mathrm{H}-$ imidazol-4-yl)- $N, N$-bis[(1-methyl- $1 H$-imidazol-2-yl)methyl]ethanamine; Higa et al., 2007\}, the copper-to-plane distances are 0.1761 (1) and 0.23918 (10) $\AA$, respectively.

The $\mathrm{Cu} 1-\mathrm{Cl} 1$ bond length is 2.2698 (10) $\AA$, being the longest in the coordination sphere. This value is in good agreement with the $\mathrm{Cu}-\mathrm{Cl}$ bond lengths of 2.2742 (11) and $2.2690(12) \AA$ reported for the complexes $[\mathrm{Cu}(\text { pmea }) \mathrm{Cl}]^{+}$ [pmea $=2$-(pyridin-2-yl)- $N, N$-bis(pyridin-2-ylmethyl)ethanamine $]$ and $[\mathrm{Cu}(\text { pmap }) \mathrm{Cl}]^{+}$[pmap $=2$-(pyridin-2-yl $)-N-(2-$ (pyridin-2-yl)ethyl)- $N$-(pyridin-2-ylmethyl)ethanamine; Schatz et al., 2001]. For the complexes [ $\mathrm{Cu}($ hismimi $\left.) \mathrm{Cl}_{2}\right]$ and $\left[\mathrm{Cu}\right.$ (hismima) $\left.\mathrm{Cl}_{2}\right]\{$ hismimi $=2-(1 H$-imidazol-4-yl $)-N-[(1-$ methyl-1H-imidazol-2-yl)methylene $]$ ethanamine; hismima = 2-(1H-imidazol-4-yl)- $N$-[(1-methyl- $1 H$-imidazol-2-yl)methyl]ethanamine; Scarpellini et al., 2003\}, the $\mathrm{Cu}-\mathrm{Cl}$ bond lengths range from 2.2882 (11) to 2.2930 (11) $\AA$ for the $\mathrm{Cl}$ atoms in the basal plane and from 2.5705 (10) to 2.5789 (10) $\AA$ for the $\mathrm{Cl}$ atoms occupying the apical position.

The $\mathrm{Cu}-\mathrm{N}_{\mathrm{Me} \text {-im }}$ bond lengths in the title compound range from 1.976 (3) to 2.173 (3) $\AA$, the longest one being formed by the 1-methyl-imidazole nitrogen N11. For the $\mathrm{Cu}-\mathrm{N}_{\mathrm{am}}$ bond, a distance of 2.137 (3) $\AA$ was found. Similar values were reported for the 1-methyl-imidazole-containing complexes $[\mathrm{Cu}(\text { Hhis-im } 2) \mathrm{Cl}]^{+}$(Higa et al., 2007), [Cu(hismima)(his) $]^{+}$ \{hismima $=2-(1 H$-imidazol-4-yl $)-N$-[(1-methyl- $1 H$-imidazol-2yl)methyl]ethanamine; his = histamine; Scarpellini et al., 2001\}, $\left.\left[\mathrm{Cu}_{2} \text { (hismima }\right)_{2} \mathrm{Cl}_{2}\right]_{2}{ }^{2+}$ (Scarpellini et al., 2004b), [Cu(pymimi) $\left.\mathrm{Cl}_{2}\right]$ and $\left[\mathrm{Cu}\right.$ (pymima) $\left.\mathrm{Cl}_{2}\right]$ \{pymimi $=$ [2-(pyridyl-2-yl $)$ ethyl][(1-methylimidazol-2-yl)methyl $]$ imine; pymima $=[2-($ pyridyl-2-yl)ethyl][(1-methylimidazol-2-yl)methyl]amine; Ferre et al., 2017\}.

\section{Supramolecular features}

Intermolecular contacts in the title compound occur through $\pi-\pi$ stacking interactions (Fig. 2) involving two 1-methyl-imidazole rings (N21/C22/N23/C25/C26 and N31/ $\mathrm{C} 32 / \mathrm{N} 33 / \mathrm{C} 35 / \mathrm{C} 36)$, forming chains that propagate parallel to the $a$ axis. The intercentroid distances are 3.690 (2) and 3.761 (2) $\AA$, the centroid-to-plane distances are 3.4719 (15) and 3.6240 (15) $\AA$, and the parallel shifts are 1.250 (6) and 1.008 (7) ̊. 

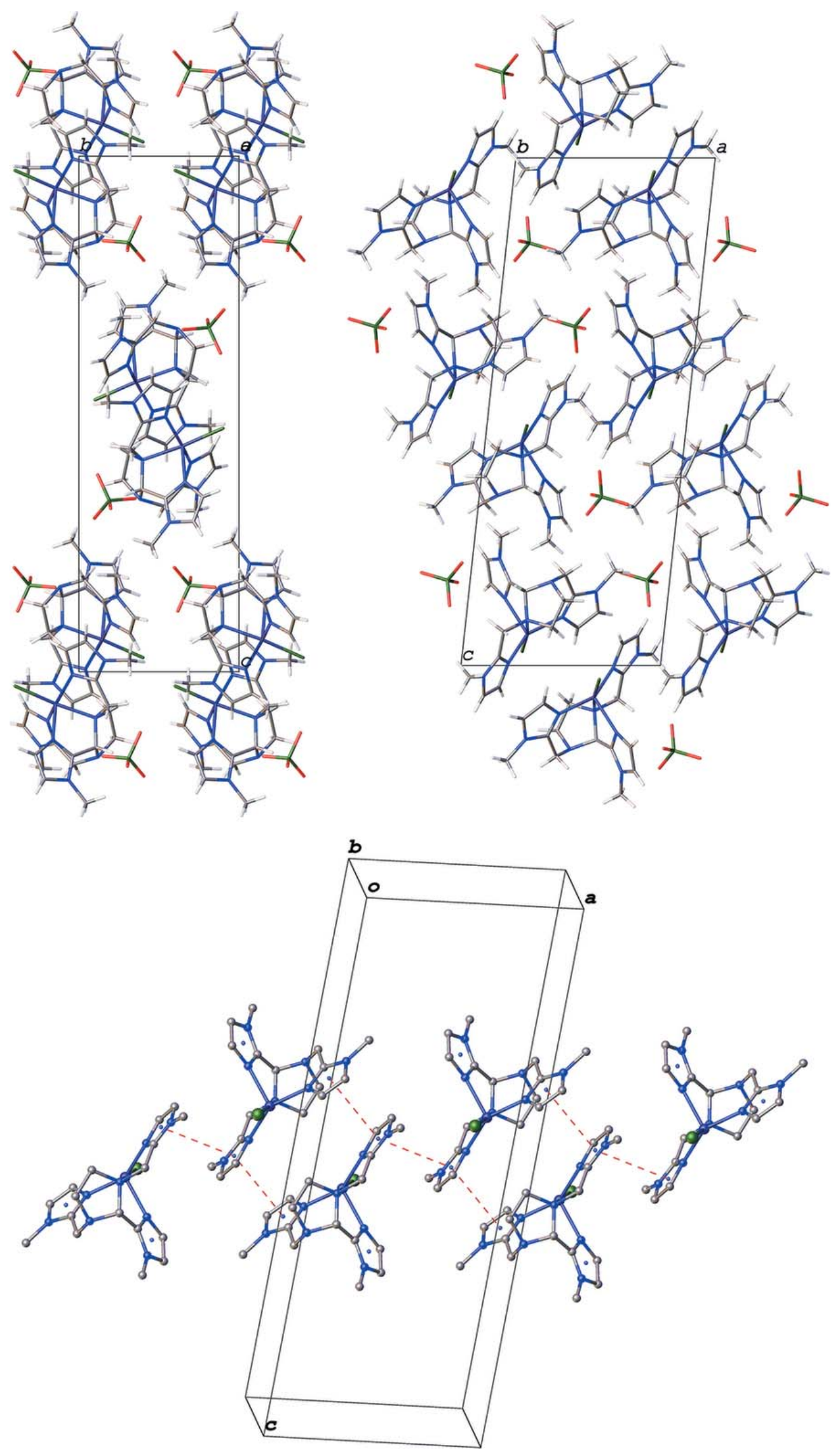

Figure 2

Crystal packing (viewed perpendicular to (100), top left, and (010), top right) and intermolecular $\pi-\pi$ stacking interactions (dashed lines, bottom) in the structure of the title compound. 


\section{Features of related complexes}

In pentacoordinated copper(II) complexes containing tripodal $\mathrm{N}_{4}$ donor ligands similar to the title compound, the $\mathrm{Cu}-\mathrm{Cl}$ bond length seems to be directly related to the type and degree of geometry distortion around the metal centre. In complexes exhibiting a square-pyramidal geometry, as in the title compound, the $\mathrm{Cu}-\mathrm{Cl}$ bond length has a range of 2.27$2.29 \AA$. For complexes in a trigonal-bipyramidal geometry, the $\mathrm{Cu}-\mathrm{Cl}$ distance is around $2.23 \AA$ (Karlin et al., 1982; Oberhausen et al., 1990; Wang et al., 1995). This difference may be related to the ligand spatial orientation, resulting from the geometric arrangements around the metal centre. The trigonal-bipyramidal geometry imposes a vertical positioning of the coordinated ligand rings parallel to the axial direction, which minimizes the repulsion between the electronic clouds of the chloride ion and the tripodal ligand. This arrangement allows a greater approach of the chloride ion to the metal centre and consequently a shorter bond distance. In the case of complexes in a square-pyramidal geometry, the coordinated rings are oriented parallel to the basal plane, increasing the chloride/ligand repulsion effect, which makes the $\mathrm{Cu}-\mathrm{Cl}$ bond more elongated. Curiously, copper complexes in both geometries with tripodal ligands showing steric hindrance exhibit intermediate $\mathrm{Cu}-\mathrm{Cl}$ bond distances among those found for complexes with non-hindered ligands on squarepyramidal and trigonal-bipyramidal geometries, indicating a balance of repulsive and stabilizing chloride/ligand interactions that is geometry independent (Wei et al., 1994; Jitsukawa et al., 2001).

\section{Synthesis and crystallization}

2,2'-\{[2-(1-Methyl-1H-imidazol-2-yl)imidazolidine-1,3-diyl]bis(methylene)\}bis(1-methyl-1H-imidazole), $\boldsymbol{L}$ : The new ligand $\mathbf{L}$ was synthesized by condensation reaction between $N^{1}, N^{2}$-bis[(1-methyl-1H-imidazol-2-yl)methyl]ethane-1,2-diamine (Neves et al., 1997) (1.8939 g, $7.63 \mathrm{mmol})$ and 1-methyl2-imidazolecarboxaldehyde $(0.8401 \mathrm{~g}, 7.63 \mathrm{mmol})$ in ethanolic media $(40 \mathrm{ml})$. The reaction mixture was stirred for $24 \mathrm{~h}$ at room temperature, when the solvent was removed by rotaevaporation. To the resulting white solid, $40 \mathrm{ml}$ of ethyl ether were added, and the mixture was stirred at room temperature for $24 \mathrm{~h}$. After removal of the solvent under reduced pressure, the resulting white solid was recrystallized from acetone. Yield after recrystallization: $2.4 \mathrm{~g} \quad(92 \%) .{ }^{1} \mathrm{H} \quad \mathrm{NMR} \quad(500 \mathrm{MHz}$, DMSO) $\delta 7.16(d, J=0.8 \mathrm{~Hz}, 1 \mathrm{H}), 7.01(d, J=1.1 \mathrm{~Hz}, 2 \mathrm{H}), 6.84$ $(d, J=1.1 \mathrm{~Hz}, 1 \mathrm{H}), 6.73(d, J=1.2 \mathrm{~Hz}, 2 \mathrm{H}), 4.20(s, 1 \mathrm{H}), 3.74(s$, $3 \mathrm{H}), 3.63(d, J=13.5 \mathrm{~Hz}, 2 \mathrm{H}), 3.43(d, J=13.5 \mathrm{~Hz}, 2 \mathrm{H}), 3.33$ (s, $6 \mathrm{H}), 2.96-2.87(m, 2 \mathrm{H}), 2.75-2.67(m, 2 \mathrm{H})$ p.p.m. ${ }^{13} \mathrm{C} \mathrm{NMR}$ (126 MHz, DMSO) $\delta 144.89,144.45,127.16,126.75,124.13$, 122.18, 82.92, 50.45, 33.37, 32.19 p.p.m..

$[\mathbf{C u}(\boldsymbol{L}) \mathbf{C l}] \mathrm{ClO}_{4}$ : The synthesis was achieved by reacting $\mathrm{CuCl}_{2} \cdot 2 \mathrm{H}_{2} \mathrm{O}(0.1708 \mathrm{~g}, 1 \mathrm{mmol})$ and the ligand $\mathbf{L}(0.3403 \mathrm{~g}$, $1 \mathrm{mmol}$ ) in ethanolic media, at room temperature. Recrystallization of the obtained amorphous green solid in acetonitrile solution at room temperature yielded $0.152 \mathrm{~g}(28 \%)$ of
Table 1

Experimental details.

\begin{tabular}{|c|c|}
\hline \multicolumn{2}{|l|}{ Crystal data } \\
\hline Chemical formula & {$\left[\mathrm{CuCl}\left(\mathrm{C}_{17} \mathrm{H}_{24} \mathrm{~N}_{8}\right)\right] \mathrm{ClO}_{4}$} \\
\hline$M_{\mathrm{r}}$ & 538.88 \\
\hline Crystal system, space group & Monoclinic, $P 2_{1} / n$ \\
\hline Temperature $(\mathrm{K})$ & 288 \\
\hline$a, b, c(\AA)$ & $10.2904(4), 8.1336(3), 26.317(1)$ \\
\hline$\beta\left(^{\circ}\right)$ & $96.170(1)$ \\
\hline$V\left(\AA^{3}\right)$ & $2189.92(14)$ \\
\hline$Z$ & 4 \\
\hline Radiation type & Mo $K \alpha$ \\
\hline$\mu\left(\mathrm{mm}^{-1}\right)$ & 1.29 \\
\hline Crystal size $(\mathrm{mm})$ & $0.25 \times 0.16 \times 0.09$ \\
\hline \multicolumn{2}{|l|}{ Data collection } \\
\hline Diffractometer & Bruker D8 Venture \\
\hline Absorption correction & $\begin{array}{l}\text { Multi-scan (SADABS; Bruker, } \\
\text { 2015) }\end{array}$ \\
\hline$T_{\min }, T_{\max }$ & $0.656,0.745$ \\
\hline $\begin{array}{l}\text { No. of measured, independent and } \\
\text { observed }[I>2 \sigma(I)] \text { reflections }\end{array}$ & $41042,4471,3704$ \\
\hline$R_{\text {int }}$ & 0.069 \\
\hline$(\sin \theta / \lambda)_{\max }\left(\AA^{-1}\right)$ & 0.625 \\
\hline \multicolumn{2}{|l|}{ Refinement } \\
\hline$R\left[F^{2}>2 \sigma\left(F^{2}\right)\right], w R\left(F^{2}\right), S$ & $0.050,0.102,1.12$ \\
\hline No. of reflections & 4471 \\
\hline No. of parameters & 319 \\
\hline No. of restraints & 63 \\
\hline $\mathrm{H}$-atom treatment & $\mathrm{H}$-atom parameters constrained \\
\hline$\Delta \rho_{\max }, \Delta \rho_{\min }\left(\mathrm{e} \AA^{-3}\right)$ & $0.52,-0.53$ \\
\hline
\end{tabular}

Computer programs: APEX2 (Bruker, 2015), SAINT (Bruker, 2015, SHELXT (Sheldrick, 2015a), SHELXL (Sheldrick, 2015b) and OLEX2 (Dolomanov et al., 2009).

green single crystals after one day. IR $\left(\mathrm{cm}^{-1}, \mathrm{KBr}\right): 3460(v$ $\mathrm{O}-\mathrm{H}), 3160-3130$ ( $\left.v \mathrm{C}-\mathrm{H}_{\text {arom }}\right), 2972-2854\left(v \mathrm{C}-\mathrm{H}_{\mathrm{ali}}\right), 1636-$ $1419\left(v \mathrm{C}=\mathrm{N} / \mathrm{C}=\mathrm{C}_{\text {ring }}\right), 1285\left(v C-\mathrm{N}_{\text {amine }}\right), 1096 / 623(v \mathrm{Cl}-$ O), $771\left(\delta \mathrm{C}-\mathrm{H}_{\text {arom }}\right), 502\left(v \mathrm{Cu}-\mathrm{N}_{\text {amine }}\right), 291(v \mathrm{Cu}-\mathrm{Cl})$.

\section{Refinement}

Crystal data, data collection and structure refinement details are summarized in Table 1 . The perchlorate anion is rotationally disordered over two orientations sharing the $\mathrm{O} 1$ oxygen atom with site occupancy factors of 0.5 . The two disordered positions were refined by applying SADI restraints on the $\mathrm{Cl}-\mathrm{O}$ bond lengths and $\mathrm{O} \cdots \mathrm{O}$ separations. The $U_{\mathrm{ij}}$ parameters of the $\mathrm{Cl} 2$ atom were restrained to an approximate isotropic behaviour.

\section{Acknowledgements}

The authors thank the Laboratório Multiusuário de Difração de Raios $X$ da Universidade Federal Fluminense (LDRX/ UFF) for the use of laboratory facilities.

\section{Funding information}

Funding for this research was provided by: Conselho Nacional de Desenvolvimento Científico e Tecnológico (scholarship No. 141341/2013-0 to D. da Silva Padilha); Coordenação de Aperfeiçoamento de Pessoal de Nível Superior (Financial Code No. 001); Fundação Carlos Chagas Filho de Amparo à 
Pesquisa do Estado do Rio de Janeiro (grant Nos. E-26/ 111.177/2011 and E-26/110.157/2014 to M. Scarpellini).

\section{References}

Bruker (2015). APEX2, SAINT and SADABS. Bruker AXS Inc., Madison, Wisconsin, USA.

Cisnetti, F., Lefèvre, A. S., Guillot, R., Lambert, F., Blain, G., Anxolabéhère-Mallart, E. \& Policar, C. (2007). Eur. J. Inorg. Chem. pp. 4472-4480.

Dolomanov, O. V., Bourhis, L. J., Gildea, R. J., Howard, J. A. K. \& Puschmann, H. (2009). J. Appl. Cryst. 42, 339-341.

Du, J., Wang, J., Ji, L., Xu, X. \& Chen, Z. (2016). Appl. Mater. Interfaces, 8, 30205-30211.

Elwell, C. E., Gagnon, N. L., Neisen, B. D., Dhar, D., Spaeth, A. D., Yee, G. M. \& Tolman, W. B. (2017). Chem. Rev. 117, 2059-2107.

Ferre, F. T., Resende, J. A. L. C., Schultz, J., Mangrich, A. S., Faria, R. B., Rocha, A. B. \& Scarpellini, M. (2017). Polyhedron, 123, $293-$ 304.

Garcia, L., Cisnetti, F., Gillet, N., Guillot, R., Aumont-Nicaise, M., Piquemal, J. P., Desmadril, M., Lambert, F. \& Policar, C. (2015). J. Am. Chem. Soc. 137, 1141-1146.

Haddad, A. Z., Cronin, S. P., Mashuta, M. S., Buchanan, R. M. \& Grapperhaus, C. A. (2017). Inorg. Chem. 56, 11254-11265.

Higa, T., Moriya, M., Shimazaki, Y., Yajima, T., Tani, F., Karasawa, S., Nakano, M., Naruta, Y. \& Yamauchi, O. (2007). Inorg. Chim. Acta, 360, 3304-3313.

Jitsukawa, K., Harata, M., Arii, H., Sakurai, H. \& Masuda, H. (2001). Inorg. Chim. Acta, 324, 108-116.

Karlin, K. D., Hayes, J. C., Juen, S., Hutchinson, J. P. \& Zubieta, J. (1982). Inorg. Chem. 21, 4106-4108.
Khusnutdinova, D., Wadsworth, B. L., Flores, M., Beiler, A. M., Reyes Cruz, E. A., Zenkov, Y. \& Moore, G. F. (2018). ACS Catal. 8, 98889898.

Neves, A., Tamanini, M., Correia, V. R. \& Vencato, I. (1997). J. Braz. Chem. Soc. 8, 519-522.

Oberhausen, K. J., O'brien, R. J., Richardson, J. F. \& Buchanan, R. M. (1990). Inorg. Chim. Acta, 173, 145-154.

Scarpellini, M., Neves, A., Bortoluzzi, A. J. \& Joussef, A. C. (2001). Acta Cryst. C57, 356-358.

Scarpellini, M., Neves, A., Castellano, E. E., de Almeida Neves, E. F. \& Franco, D. W. (2004a). Polyhedron, 23, 511-518.

Scarpellini, M., Neves, A., Castellano, E. E. \& Franco, D. W. (2004b). J. Mol. Struct. 694, 193-198.

Scarpellini, M., Neves, A., Hörner, R., Bortoluzzi, A. J., Szpoganics, B., Zucco, C., Nome Silva, R. A., Drago, V., Mangrich, A. S., Ortiz, W. A., Passos, W. A. C., de Oliveira, M. C. B. \& Terenzi, H. (2003). Inorg. Chem. 42, 8353-8365.

Schatz, M., Becker, M., Thaler, F., Hampel, F., Schindler, S., Jacobson, R. R., Tyeklár, Z., Murthy, N. N., Ghosh, P., Chen, Q., Zubieta, J. \& Karlin, K. D. (2001). Inorg. Chem. 40, 2312-2322.

Sheldrick, G. M. (2015a). Acta Cryst. A71, 3-8.

Sheldrick, G. M. (2015b). Acta Cryst. C71, 3-8.

Stephanos, J. J. \& Addison, A. W. (2014). Chemistry of Metalloproteins: Problems and Solutions in Bioinorganic Chemistry, pp. 95146. Hoboken, New Jersey: John Wiley \& Sons, Inc.

Wang, J., Mashuta, M. S., Richardson, J. F. \& Buchanan, R. M. (1995). Acta Cryst. C51, 50-52.

Wei, N., Murthy, N. N. \& Karlin, K. D. (1994). Inorg. Chem. 33, $6093-$ 6100.

Zhang, P., Wang, M., Chen, H., Liang, Y., Sun, J. \& Sun, L. (2016). Adv. Energy Mater. 6, 1-9.

Zhang, P., Wang, M., Yang, Y., Yao, T. \& Sun, L. (2014). Angew. Chem. Int. Ed. 53, 13803-13807. 


\section{supporting information}

Acta Cryst. (2019). E75, 547-551 [https://doi.org/10.1107/S2056989019004055]

Chlorido(2,2'-\{[2-(1-methyl-1H-imidazol-2-yl- $\left.\kappa N^{3}\right)$ imidazolidine-1,3-diyl$\kappa N]$ bis(methylene)\}bis(1-methyl-1H-imidazole- $\left.\kappa N^{3}\right)$ )copper(II) perchlorate

\section{Diego da Silva Padilha and Marciela Scarpellini}

\section{Computing details}

Data collection: APEX2 (Bruker, 2015); cell refinement: SAINT (Bruker, 2015); data reduction: SAINT (Bruker, 2015); program(s) used to solve structure: SHELXT (Sheldrick, 2015a); program(s) used to refine structure: SHELXL (Sheldrick, 2015b); molecular graphics: OLEX2 (Dolomanov et al., 2009); software used to prepare material for publication: OLEX2 (Dolomanov et al., 2009).

Chlorido(2,2'-\{[2-(1-methyl-1H-imidazol-2-yl- $\left.\kappa N^{3}\right)$ imidazolidine-1,3-diyl- $\left.\kappa N\right]$ bis(methylene)\}bis(1-methyl-1Himidazole- $\left.\kappa N^{3}\right)$ )copper(II) perchlorate

\section{Crystal data}

$\left[\mathrm{CuCl}\left(\mathrm{C}_{17} \mathrm{H}_{24} \mathrm{~N}_{8}\right)\right] \mathrm{ClO}_{4}$

$M_{r}=538.88$

Monoclinic, $P 2_{1} / n$

$a=10.2904(4) \AA$

$b=8.1336(3) \AA$

$c=26.317(1) \AA$

$\beta=96.170(1)^{\circ}$

$V=2189.92(14) \AA^{3}$

$Z=4$

\section{Data collection}

Bruker D8 Venture diffractometer

$\varphi$ and $\omega$ scans

Absorption correction: multi-scan

(SADABS; Bruker, 2015)

$T_{\min }=0.656, T_{\max }=0.745$

41042 measured reflections

\section{Refinement}

Refinement on $F^{2}$

Least-squares matrix: full

$R\left[F^{2}>2 \sigma\left(F^{2}\right)\right]=0.050$

$w R\left(F^{2}\right)=0.102$

$S=1.12$

4471 reflections

319 parameters

63 restraints

Primary atom site location: dual
$F(000)=1108$

$D_{\mathrm{x}}=1.634 \mathrm{Mg} \mathrm{m}^{-3}$

Mo $K \alpha$ radiation, $\lambda=0.71073 \AA$

Cell parameters from 117 reflections

$\theta=2.9-22.7^{\circ}$

$\mu=1.29 \mathrm{~mm}^{-1}$

$T=288 \mathrm{~K}$

Block, clear light green

$0.25 \times 0.16 \times 0.09 \mathrm{~mm}$

4471 independent reflections

3704 reflections with $I>2 \sigma(I)$

$R_{\text {int }}=0.069$

$\theta_{\max }=26.4^{\circ}, \theta_{\min }=2.2^{\circ}$

$h=-12 \rightarrow 12$

$k=-10 \rightarrow 10$

$l=-32 \rightarrow 32$

Hydrogen site location: inferred from neighbouring sites

$\mathrm{H}$-atom parameters constrained

$w=1 /\left[\sigma^{2}\left(F_{\mathrm{o}}{ }^{2}\right)+(0.0274 P)^{2}+4.7286 P\right]$

where $P=\left(F_{\mathrm{o}}{ }^{2}+2 F_{\mathrm{c}}{ }^{2}\right) / 3$

$(\Delta / \sigma)_{\max }=0.001$

$\Delta \rho_{\max }=0.52 \mathrm{e}^{-3}$

$\Delta \rho_{\min }=-0.53$ e $\AA^{-3}$ 


\section{Special details}

Geometry. All esds (except the esd in the dihedral angle between two 1.s. planes) are estimated using the full covariance matrix. The cell esds are taken into account individually in the estimation of esds in distances, angles and torsion angles; correlations between esds in cell parameters are only used when they are defined by crystal symmetry. An approximate (isotropic) treatment of cell esds is used for estimating esds involving l.s. planes.

Refinement. All $\mathrm{H}$ atoms were placed geometrically and refined using a riding atom approximation, with $\mathrm{C}-\mathrm{H}=0.93-$ $0.98 \AA$, and with $U_{\text {iso }}(\mathrm{H})=1.2 U_{\text {eq }}(\mathrm{C})$ or $1.5 U_{\text {eq }}(\mathrm{C})$ for methyl $\mathrm{H}$ atoms. A rotating model was used for the methyl groups.

Fractional atomic coordinates and isotropic or equivalent isotropic displacement parameters $\left(\AA^{2}\right)$

\begin{tabular}{|c|c|c|c|c|c|}
\hline & $x$ & $y$ & $z$ & $U_{\text {iso }} * / U_{\text {eq }}$ & Occ. $(<1)$ \\
\hline $\mathrm{Cu} 1$ & 0.18398 (4) & $0.35492(5)$ & $0.56226(2)$ & $0.02658(12)$ & \\
\hline $\mathrm{Cl} 2$ & $0.61349(10)$ & $0.81015(12)$ & $0.66768(4)$ & $0.0394(2)$ & \\
\hline $\mathrm{Cl1}$ & $0.20109(10)$ & $0.10125(11)$ & $0.52765(4)$ & $0.0442(3)$ & \\
\hline $\mathrm{N} 21$ & $0.2633(3)$ & 0.4598 (3) & $0.50530(11)$ & $0.0276(6)$ & \\
\hline N1 & $0.1935(3)$ & $0.6031(3)$ & $0.58904(10)$ & $0.0237(6)$ & \\
\hline N31 & 0.0181 (3) & $0.3032(4)$ & $0.59528(11)$ & $0.0298(6)$ & \\
\hline $\mathrm{N} 23$ & $0.3807(3)$ & 0.6599 (4) & $0.47829(11)$ & $0.0293(6)$ & \\
\hline N11 & $0.3159(3)$ & $0.3367(4)$ & $0.63224(12)$ & $0.0324(7)$ & \\
\hline N33 & -0.1361 & 0.2601 (4) & $0.64552(11)$ & $0.0332(7)$ & \\
\hline N13 & $0.3635(3)$ & 0.4347 (4) & $0.70991(11)$ & $0.0362(7)$ & \\
\hline N4 & 0.0918 (3) & $0.6184(4)$ & $0.66510(11)$ & $0.0331(7)$ & \\
\hline $\mathrm{C} 12$ & $0.2988(3)$ & $0.4602(4)$ & $0.66327(13)$ & $0.0283(7)$ & \\
\hline $\mathrm{C} 26$ & $0.2978(3)$ & $0.4181(5)$ & 0.45779 (13) & $0.0319(8)$ & \\
\hline $\mathrm{H} 26$ & 0.275354 & 0.321232 & 0.440245 & $0.038 *$ & \\
\hline $\mathrm{C} 22$ & $0.3146(3)$ & 0.6059 (4) & $0.51634(12)$ & $0.0241(7)$ & \\
\hline $\mathrm{C} 5$ & $0.2183(3)$ & 0.6065 (4) & $0.64627(12)$ & $0.0267(7)$ & \\
\hline H5 & 0.268323 & 0.705847 & 0.656460 & $0.032^{*}$ & \\
\hline $\mathrm{C} 32$ & $-0.0283(3)$ & $0.3493(5)$ & $0.63838(13)$ & $0.0307(8)$ & \\
\hline C6 & $0.3044(3)$ & $0.6840(4)$ & $0.56629(13)$ & $0.0286(8)$ & \\
\hline H6A & 0.287929 & 0.800888 & 0.561970 & $0.034 *$ & \\
\hline H6B & 0.385168 & 0.669535 & 0.588489 & $0.034^{*}$ & \\
\hline $\mathrm{C} 36$ & $-0.0650(3)$ & 0.1818 (4) & $0.57440(15)$ & $0.0336(8)$ & \\
\hline H36 & -0.057209 & 0.127441 & 0.543794 & $0.040 *$ & \\
\hline $\mathrm{C} 25$ & $0.3696(4)$ & $0.5416(5)$ & $0.44102(14)$ & $0.0341(8)$ & \\
\hline $\mathrm{H} 25$ & 0.405045 & 0.545645 & 0.409984 & $0.041^{*}$ & \\
\hline $\mathrm{C} 35$ & $-0.1586(3)$ & $0.1546(5)$ & $0.60512(15)$ & $0.0388(9)$ & \\
\hline H35 & -0.226056 & 0.078382 & 0.599912 & $0.047 *$ & \\
\hline $\mathrm{C} 7$ & $0.0270(4)$ & $0.4682(5)$ & $0.67877(14)$ & $0.0388(9)$ & \\
\hline H7A & -0.044043 & 0.500341 & 0.698061 & $0.047^{*}$ & \\
\hline H7B & 0.089098 & 0.407663 & 0.702043 & $0.047^{*}$ & \\
\hline $\mathrm{C} 2$ & $0.0650(3)$ & $0.6893(4)$ & $0.57597(14)$ & $0.0328(8)$ & \\
\hline $\mathrm{H} 2 \mathrm{~A}$ & 0.003612 & 0.619174 & 0.555523 & $0.039 *$ & \\
\hline $\mathrm{H} 2 \mathrm{~B}$ & 0.076571 & 0.790207 & 0.557356 & $0.039^{*}$ & \\
\hline $\mathrm{C} 24$ & $0.4503(4)$ & $0.8151(5)$ & $0.47750(16)$ & $0.0414(10)$ & \\
\hline $\mathrm{H} 24 \mathrm{~A}$ & 0.390577 & 0.904225 & 0.480668 & $0.062 *$ & \\
\hline H24B & 0.488065 & 0.825186 & 0.445837 & $0.062 *$ & \\
\hline $\mathrm{H} 24 \mathrm{C}$ & 0.518329 & 0.818347 & 0.505466 & $0.062 *$ & \\
\hline
\end{tabular}




$\begin{array}{lllll}\text { C16 } & 0.3943(4) & 0.2272(5) & 0.66066(16) & 0.0407(9) \\ \text { H16 } & 0.422015 & 0.126906 & 0.648747 & 0.049^{*} \\ \text { C3 } & 0.0176(4) & 0.7253(5) & 0.62797(15) & 0.0437(10) \\ \text { H3A } & 0.032731 & 0.839800 & 0.637102 & 0.052^{*} \\ \text { H3B } & -0.075173 & 0.702826 & 0.626991 & 0.052^{*} \\ \text { C15 } & 0.4254(4) & 0.2850(5) & 0.70806(16) & 0.0458(10) \\ \text { H15 } & 0.478094 & 0.234306 & 0.734412 & 0.055^{*} \\ \text { C34 } & -0.2202(4) & 0.2733(6) & 0.68726(17) & 0.0541(12) \\ \text { H34A } & -0.169792 & 0.249093 & 0.719228 & 0.081^{*} \\ \text { H34B } & -0.291094 & 0.196567 & 0.681554 & 0.081^{*} \\ \text { H34C } & -0.254383 & 0.382966 & 0.688193 & 0.081^{*} \\ \text { C14 } & 0.3707(5) & 0.5456(6) & 0.75403(16) & 0.0577(12) \\ \text { H14A } & 0.380595 & 0.656728 & 0.742796 & 0.087^{*} \\ \text { H14B } & 0.444214 & 0.516161 & 0.777864 & 0.087^{*} \\ \text { H14C } & 0.291865 & 0.536478 & 0.770330 & 0.087^{*} \\ \text { O1 } & 0.5896(4) & 0.8829(4) & 0.61817(11) & 0.0659(10) \\ \text { O2 } & 0.551(2) & 0.904(3) & 0.7022(7) & 0.089(6) \\ \text { O3 } & 0.5625(15) & 0.6503(12) & 0.6636(6) & 0.074(4) \\ \text { O4 } & 0.7476(8) & 0.803(3) & 0.6829(7) & 0.093(6) \\ \text { O4' } & 0.7405(11) & 0.851(3) & 0.6894(7) & 0.109(7) \\ \text { O3' } & 0.604(2) & 0.6372(13) & 0.6674(8) & 0.124(8) \\ \text { O2' } & 0.526(2) & 0.870(3) & 0.7006(9) & 0.094(7)\end{array}$

Atomic displacement parameters $\left(\AA^{2}\right)$

\begin{tabular}{lllllll}
\hline & $U^{11}$ & $U^{22}$ & $U^{33}$ & $U^{12}$ & $U^{13}$ & $U^{23}$ \\
\hline $\mathrm{Cu} 1$ & $0.0292(2)$ & $0.0192(2)$ & $0.0325(2)$ & $-0.00422(17)$ & $0.00912(17)$ & $-0.00224(18)$ \\
$\mathrm{C} 12$ & $0.0445(5)$ & $0.0387(5)$ & $0.0368(5)$ & $-0.0036(4)$ & $0.0122(4)$ & $-0.0021(4)$ \\
$\mathrm{C} 11$ & $0.0441(5)$ & $0.0221(4)$ & $0.0698(7)$ & $-0.0053(4)$ & $0.0219(5)$ & $-0.0113(4)$ \\
$\mathrm{N} 21$ & $0.0282(15)$ & $0.0246(15)$ & $0.0307(16)$ & $-0.0030(12)$ & $0.0062(12)$ & $-0.0021(12)$ \\
$\mathrm{N} 1$ & $0.0239(14)$ & $0.0234(14)$ & $0.0240(14)$ & $0.0002(11)$ & $0.0038(11)$ & $0.0010(11)$ \\
$\mathrm{N} 31$ & $0.0260(15)$ & $0.0310(16)$ & $0.0329(16)$ & $-0.0058(12)$ & $0.0058(12)$ & $-0.0013(13)$ \\
$\mathrm{N} 23$ & $0.0258(14)$ & $0.0281(15)$ & $0.0350(16)$ & $0.0026(12)$ & $0.0077(12)$ & $0.0080(13)$ \\
$\mathrm{N} 11$ & $0.0320(16)$ & $0.0256(15)$ & $0.0397(17)$ & $0.0030(13)$ & $0.0041(13)$ & $0.0037(14)$ \\
$\mathrm{N} 33$ & $0.0213(15)$ & $0.0458(19)$ & $0.0336(17)$ & $-0.0043(13)$ & $0.0073(12)$ & $0.0035(15)$ \\
$\mathrm{N} 13$ & $0.0349(17)$ & $0.0458(19)$ & $0.0278(16)$ & $-0.0012(14)$ & $0.0028(13)$ & $0.0076(14)$ \\
$\mathrm{N} 4$ & $0.0322(16)$ & $0.0355(17)$ & $0.0328(16)$ & $0.0020(13)$ & $0.0090(13)$ & $-0.0078(14)$ \\
$\mathrm{C} 12$ & $0.0237(17)$ & $0.0317(19)$ & $0.0296(19)$ & $-0.0019(14)$ & $0.0034(14)$ & $0.0039(15)$ \\
$\mathrm{C} 26$ & $0.0322(19)$ & $0.0334(19)$ & $0.0302(19)$ & $-0.0010(15)$ & $0.0036(15)$ & $-0.0064(16)$ \\
$\mathrm{C} 22$ & $0.0238(16)$ & $0.0204(16)$ & $0.0279(17)$ & $-0.0002(13)$ & $0.0024(13)$ & $0.0020(13)$ \\
$\mathrm{C} 5$ & $0.0280(17)$ & $0.0263(18)$ & $0.0256(17)$ & $-0.0013(14)$ & $0.0016(14)$ & $-0.0033(14)$ \\
$\mathrm{C} 32$ & $0.0244(17)$ & $0.038(2)$ & $0.0307(18)$ & $-0.0017(15)$ & $0.0057(14)$ & $0.0013(16)$ \\
$\mathrm{C} 6$ & $0.0309(18)$ & $0.0213(17)$ & $0.0339(19)$ & $-0.0068(14)$ & $0.0055(15)$ & $-0.0012(14)$ \\
$\mathrm{C} 36$ & $0.0274(18)$ & $0.034(2)$ & $0.040(2)$ & $-0.0056(15)$ & $0.0046(15)$ & $-0.0016(16)$ \\
$\mathrm{C} 25$ & $0.0341(19)$ & $0.042(2)$ & $0.0278(19)$ & $0.0046(16)$ & $0.0088(15)$ & $0.0031(17)$ \\
$\mathrm{C} 35$ & $0.0263(18)$ & $0.044(2)$ & $0.045(2)$ & $-0.0093(17)$ & $-0.0015(16)$ & $0.0039(19)$ \\
$\mathrm{C} 7$ & $0.034(2)$ & $0.052(2)$ & $0.033(2)$ & $-0.0089(18)$ & $0.0145(16)$ & $-0.0053(18)$ \\
$\mathrm{C} 2$ & $0.0327(19)$ & $0.0305(19)$ & $0.034(2)$ & $0.0044(15)$ & $-0.0007(15)$ & $0.0015(16)$ \\
& & & & & &
\end{tabular}




\begin{tabular}{lllllll}
\hline & & & & & \\
$\mathrm{C} 24$ & $0.036(2)$ & $0.036(2)$ & $0.054(3)$ & $-0.0064(17)$ & $0.0154(18)$ & $0.0106(19)$ \\
$\mathrm{C} 16$ & $0.038(2)$ & $0.034(2)$ & $0.050(2)$ & $0.0086(17)$ & $0.0088(18)$ & $0.0113(19)$ \\
$\mathrm{C} 3$ & $0.040(2)$ & $0.047(2)$ & $0.044(2)$ & $0.0149(19)$ & $0.0039(18)$ & $-0.009(2)$ \\
$\mathrm{C} 15$ & $0.037(2)$ & $0.056(3)$ & $0.044(2)$ & $0.011(2)$ & $0.0034(18)$ & $0.024(2)$ \\
$\mathrm{C} 34$ & $0.034(2)$ & $0.082(3)$ & $0.050(3)$ & $-0.011(2)$ & $0.0230(19)$ & $-0.001(2)$ \\
$\mathrm{C} 14$ & $0.067(3)$ & $0.074(3)$ & $0.030(2)$ & $0.003(3)$ & $-0.003(2)$ & $-0.002(2)$ \\
O1 & $0.088(3)$ & $0.072(2)$ & $0.0390(17)$ & $-0.0155(19)$ & $0.0133(16)$ & $0.0106(16)$ \\
O2 & $0.141(15)$ & $0.087(9)$ & $0.041(7)$ & $0.065(10)$ & $0.020(8)$ & $-0.001(6)$ \\
O3 & $0.093(7)$ & $0.060(8)$ & $0.066(7)$ & $-0.046(6)$ & $-0.006(5)$ & $0.026(6)$ \\
O4 & $0.023(5)$ & $0.143(13)$ & $0.112(11)$ & $0.011(6)$ & $0.010(5)$ & $-0.008(8)$ \\
O4' & $0.098(11)$ & $0.158(16)$ & $0.066(8)$ & $-0.059(10)$ & $-0.012(7)$ & $0.035(9)$ \\
O3 $^{\prime}$ & $0.23(2)$ & $0.041(7)$ & $0.106(12)$ & $0.035(8)$ & $0.036(13)$ & $-0.011(6)$ \\
O2 $^{\prime}$ & $0.097(9)$ & $0.111(14)$ & $0.085(12)$ & $0.045(9)$ & $0.065(9)$ & $0.029(8)$ \\
\end{tabular}

Geometric parameters $\left(\AA,{ }^{\circ}\right)$

\begin{tabular}{|c|c|c|c|}
\hline $\mathrm{Cu} 1-\mathrm{Cl1}$ & $2.2698(10)$ & $\mathrm{N} 4-\mathrm{C} 3$ & $1.461(5)$ \\
\hline $\mathrm{Cu} 1-\mathrm{N} 21$ & $1.976(3)$ & $\mathrm{C} 12-\mathrm{C} 5$ & $1.491(5)$ \\
\hline $\mathrm{Cu} 1-\mathrm{N} 1$ & $2.137(3)$ & $\mathrm{C} 26-\mathrm{H} 26$ & 0.9300 \\
\hline $\mathrm{Cu} 1-\mathrm{N} 31$ & $2.041(3)$ & $\mathrm{C} 26-\mathrm{C} 25$ & $1.349(5)$ \\
\hline $\mathrm{Cu} 1-\mathrm{N} 11$ & $2.173(3)$ & $\mathrm{C} 22-\mathrm{C} 6$ & $1.474(5)$ \\
\hline $\mathrm{Cl} 2-\mathrm{O} 1$ & 1.428 & $\mathrm{C} 5-\mathrm{H} 5$ & 0.9800 \\
\hline $\mathrm{Cl} 2-\mathrm{O} 2$ & $1.395(10)$ & $\mathrm{C} 32-\mathrm{C} 7$ & $1.503(5)$ \\
\hline $\mathrm{C} 12-\mathrm{O} 3$ & $1.402(8)$ & C6-H6A & 0.9700 \\
\hline $\mathrm{Cl} 2-\mathrm{O} 4$ & $1.396(9)$ & C6-H6B & 0.9700 \\
\hline $\mathrm{Cl} 2-\mathrm{O} 4^{\prime}$ & $1.410(11)$ & $\mathrm{C} 36-\mathrm{H} 36$ & 0.9300 \\
\hline $\mathrm{Cl} 2-\mathrm{O}^{\prime}$ & $1.410(10)$ & $\mathrm{C} 36-\mathrm{C} 35$ & $1.341(5)$ \\
\hline $\mathrm{Cl} 2-\mathrm{O} 2^{\prime}$ & $1.405(10)$ & $\mathrm{C} 25-\mathrm{H} 25$ & 0.9300 \\
\hline $\mathrm{N} 21-\mathrm{C} 26$ & $1.378(4)$ & C35-H35 & 0.9300 \\
\hline $\mathrm{N} 21-\mathrm{C} 22$ & $1.320(4)$ & $\mathrm{C} 7-\mathrm{H} 7 \mathrm{~A}$ & 0.9700 \\
\hline $\mathrm{N} 1-\mathrm{C} 5$ & $1.501(4)$ & $\mathrm{C} 7-\mathrm{H} 7 \mathrm{~B}$ & 0.9700 \\
\hline $\mathrm{N} 1-\mathrm{C} 6$ & $1.497(4)$ & $\mathrm{C} 2-\mathrm{H} 2 \mathrm{~A}$ & 0.9700 \\
\hline $\mathrm{N} 1-\mathrm{C} 2$ & $1.503(4)$ & $\mathrm{C} 2-\mathrm{H} 2 \mathrm{~B}$ & 0.9700 \\
\hline $\mathrm{N} 31-\mathrm{C} 32$ & $1.331(4)$ & $\mathrm{C} 2-\mathrm{C} 3$ & $1.530(5)$ \\
\hline $\mathrm{N} 31-\mathrm{C} 36$ & $1.381(4)$ & $\mathrm{C} 24-\mathrm{H} 24 \mathrm{~A}$ & 0.9600 \\
\hline $\mathrm{N} 23-\mathrm{C} 22$ & $1.343(4)$ & $\mathrm{C} 24-\mathrm{H} 24 \mathrm{~B}$ & 0.9600 \\
\hline $\mathrm{N} 23-\mathrm{C} 25$ & $1.370(5)$ & $\mathrm{C} 24-\mathrm{H} 24 \mathrm{C}$ & 0.9600 \\
\hline $\mathrm{N} 23-\mathrm{C} 24$ & $1.452(5)$ & $\mathrm{C} 16-\mathrm{H} 16$ & 0.9300 \\
\hline $\mathrm{N} 11-\mathrm{C} 12$ & $1.318(5)$ & $\mathrm{C} 16-\mathrm{C} 15$ & $1.339(6)$ \\
\hline $\mathrm{N} 11-\mathrm{C} 16$ & $1.368(5)$ & $\mathrm{C} 3-\mathrm{H} 3 \mathrm{~A}$ & 0.9700 \\
\hline $\mathrm{N} 33-\mathrm{C} 32$ & $1.355(4)$ & $\mathrm{C} 3-\mathrm{H} 3 \mathrm{~B}$ & 0.9700 \\
\hline $\mathrm{N} 33-\mathrm{C} 35$ & $1.366(5)$ & $\mathrm{C} 15-\mathrm{H} 15$ & 0.9300 \\
\hline N33-C34 & $1.473(4)$ & $\mathrm{C} 34-\mathrm{H} 34 \mathrm{~A}$ & 0.9600 \\
\hline $\mathrm{N} 13-\mathrm{C} 12$ & $1.348(4)$ & C34-H34B & 0.9600 \\
\hline $\mathrm{N} 13-\mathrm{C} 15$ & $1.377(5)$ & $\mathrm{C} 34-\mathrm{H} 34 \mathrm{C}$ & 0.9600 \\
\hline $\mathrm{N} 13-\mathrm{C} 14$ & $1.466(5)$ & $\mathrm{C} 14-\mathrm{H} 14 \mathrm{~A}$ & 0.9600 \\
\hline $\mathrm{N} 4-\mathrm{C} 5$ & $1.445(4)$ & $\mathrm{C} 14-\mathrm{H} 14 \mathrm{~B}$ & 0.9600 \\
\hline $\mathrm{N} 4-\mathrm{C} 7$ & $1.455(5)$ & $\mathrm{C} 14-\mathrm{H} 14 \mathrm{C}$ & 0.9600 \\
\hline
\end{tabular}




\begin{tabular}{|c|c|c|c|}
\hline $\mathrm{N} 21-\mathrm{Cu} 1-\mathrm{Cl} 1$ & $91.82(9)$ & $\mathrm{N} 1-\mathrm{C} 5-\mathrm{H} 5$ & 108.5 \\
\hline $\mathrm{N} 21-\mathrm{Cu} 1-\mathrm{N} 1$ & $80.47(11)$ & $\mathrm{N} 4-\mathrm{C} 5-\mathrm{N} 1$ & $106.5(3)$ \\
\hline $\mathrm{N} 21-\mathrm{Cu} 1-\mathrm{N} 31$ & $147.65(12)$ & $\mathrm{N} 4-\mathrm{C} 5-\mathrm{C} 12$ & $116.3(3)$ \\
\hline $\mathrm{N} 21-\mathrm{Cu} 1-\mathrm{N} 11$ & $113.64(12)$ & $\mathrm{N} 4-\mathrm{C} 5-\mathrm{H} 5$ & 108.5 \\
\hline $\mathrm{N} 1-\mathrm{Cu} 1-\mathrm{Cl1}$ & $171.21(8)$ & $\mathrm{C} 12-\mathrm{C} 5-\mathrm{N} 1$ & $108.4(3)$ \\
\hline $\mathrm{N} 1-\mathrm{Cu} 1-\mathrm{N} 11$ & $77.43(11)$ & $\mathrm{C} 12-\mathrm{C} 5-\mathrm{H} 5$ & 108.5 \\
\hline $\mathrm{N} 31-\mathrm{Cu} 1-\mathrm{Cl} 1$ & $95.04(9)$ & $\mathrm{N} 31-\mathrm{C} 32-\mathrm{N} 33$ & $110.0(3)$ \\
\hline $\mathrm{N} 31-\mathrm{Cu} 1-\mathrm{N} 1$ & $93.74(11)$ & $\mathrm{N} 31-\mathrm{C} 32-\mathrm{C} 7$ & $129.8(3)$ \\
\hline $\mathrm{N} 31-\mathrm{Cu} 1-\mathrm{N} 11$ & $95.74(11)$ & $\mathrm{N} 33-\mathrm{C} 32-\mathrm{C} 7$ & $120.0(3)$ \\
\hline $\mathrm{N} 11-\mathrm{Cu} 1-\mathrm{Cl} 1$ & $102.11(8)$ & $\mathrm{N} 1-\mathrm{C} 6-\mathrm{H} 6 \mathrm{~A}$ & 110.3 \\
\hline $\mathrm{O} 2-\mathrm{Cl} 2-\mathrm{O} 1$ & $108.7(10)$ & $\mathrm{N} 1-\mathrm{C} 6-\mathrm{H} 6 \mathrm{~B}$ & 110.3 \\
\hline $\mathrm{O} 2-\mathrm{Cl} 2-\mathrm{O} 3$ & $111.3(11)$ & $\mathrm{C} 22-\mathrm{C} 6-\mathrm{N} 1$ & $107.3(3)$ \\
\hline $\mathrm{O} 2-\mathrm{Cl} 2-\mathrm{O} 4$ & $110.3(11)$ & $\mathrm{C} 22-\mathrm{C} 6-\mathrm{H} 6 \mathrm{~A}$ & 110.3 \\
\hline $\mathrm{O} 2-\mathrm{Cl} 2-\mathrm{O}^{\prime}$ & $94.5(15)$ & $\mathrm{C} 22-\mathrm{C} 6-\mathrm{H} 6 \mathrm{~B}$ & 110.3 \\
\hline $\mathrm{O} 2-\mathrm{Cl} 2-\mathrm{O}^{\prime}$ & $120.9(15)$ & $\mathrm{H} 6 \mathrm{~A}-\mathrm{C} 6-\mathrm{H} 6 \mathrm{~B}$ & 108.5 \\
\hline $\mathrm{O} 2-\mathrm{Cl} 2-\mathrm{O} 2{ }^{\prime}$ & $16(2)$ & $\mathrm{N} 31-\mathrm{C} 36-\mathrm{H} 36$ & 125.3 \\
\hline $\mathrm{O} 3-\mathrm{Cl} 2-\mathrm{O} 1$ & $106.8(7)$ & $\mathrm{C} 35-\mathrm{C} 36-\mathrm{N} 31$ & $109.4(3)$ \\
\hline $\mathrm{O} 3-\mathrm{Cl} 2-\mathrm{O} 4^{\prime}$ & $125.1(12)$ & $\mathrm{C} 35-\mathrm{C} 36-\mathrm{H} 36$ & 125.3 \\
\hline $\mathrm{O} 3-\mathrm{Cl} 2-\mathrm{O}^{\prime}$ & $18.1(13)$ & $\mathrm{N} 23-\mathrm{C} 25-\mathrm{H} 25$ & 126.4 \\
\hline $\mathrm{O} 3-\mathrm{Cl} 2-\mathrm{O} 2{ }^{\prime}$ & $96.2(15)$ & $\mathrm{C} 26-\mathrm{C} 25-\mathrm{N} 23$ & $107.1(3)$ \\
\hline $\mathrm{O} 4-\mathrm{Cl} 2-\mathrm{O} 1$ & $110.3(9)$ & $\mathrm{C} 26-\mathrm{C} 25-\mathrm{H} 25$ & 126.4 \\
\hline $\mathrm{O} 4-\mathrm{Cl} 2-\mathrm{O} 3$ & $109.3(10)$ & $\mathrm{N} 33-\mathrm{C} 35-\mathrm{H} 35$ & 126.5 \\
\hline $\mathrm{O} 4-\mathrm{Cl} 2-\mathrm{O}^{\prime}$ & $18.1(16)$ & $\mathrm{C} 36-\mathrm{C} 35-\mathrm{N} 33$ & $107.0(3)$ \\
\hline $\mathrm{O} 4-\mathrm{Cl} 2-\mathrm{O}^{\prime}$ & $91.3(13)$ & $\mathrm{C} 36-\mathrm{C} 35-\mathrm{H} 35$ & 126.5 \\
\hline $\mathrm{O} 4-\mathrm{Cl} 2-\mathrm{O} 2^{\prime}$ & $121.4(15)$ & $\mathrm{N} 4-\mathrm{C} 7-\mathrm{C} 32$ & $120.9(3)$ \\
\hline $\mathrm{O} 4{ }^{\prime}-\mathrm{C} 2-\mathrm{O} 1$ & $109.4(7)$ & $\mathrm{N} 4-\mathrm{C} 7-\mathrm{H} 7 \mathrm{~A}$ & 107.1 \\
\hline $\mathrm{O} 3{ }^{\prime}-\mathrm{C} 2-\mathrm{O} 1$ & $113.8(9)$ & $\mathrm{N} 4-\mathrm{C} 7-\mathrm{H} 7 \mathrm{~B}$ & 107.1 \\
\hline $\mathrm{O}^{\prime}-\mathrm{Cl} 2-\mathrm{O} 4^{\prime}$ & $107.5(11)$ & $\mathrm{C} 32-\mathrm{C} 7-\mathrm{H} 7 \mathrm{~A}$ & 107.1 \\
\hline $\mathrm{O} 2{ }^{\prime}-\mathrm{Cl} 2-\mathrm{O} 1$ & $111.1(12)$ & $\mathrm{C} 32-\mathrm{C} 7-\mathrm{H} 7 \mathrm{~B}$ & 107.1 \\
\hline $\mathrm{O} 2^{\prime}-\mathrm{Cl} 2-\mathrm{O} 4^{\prime}$ & $107.4(12)$ & $\mathrm{H} 7 \mathrm{~A}-\mathrm{C} 7-\mathrm{H} 7 \mathrm{~B}$ & 106.8 \\
\hline $\mathrm{O} 2^{\prime}-\mathrm{Cl} 2-\mathrm{O}^{\prime}$ & $107.5(12)$ & $\mathrm{N} 1-\mathrm{C} 2-\mathrm{H} 2 \mathrm{~A}$ & 111.0 \\
\hline $\mathrm{C} 26-\mathrm{N} 21-\mathrm{Cu} 1$ & $138.6(2)$ & $\mathrm{N} 1-\mathrm{C} 2-\mathrm{H} 2 \mathrm{~B}$ & 111.0 \\
\hline $\mathrm{C} 22-\mathrm{N} 21-\mathrm{Cu} 1$ & $114.2(2)$ & $\mathrm{N} 1-\mathrm{C} 2-\mathrm{C} 3$ & $104.0(3)$ \\
\hline $\mathrm{C} 22-\mathrm{N} 21-\mathrm{C} 26$ & $106.5(3)$ & $\mathrm{H} 2 \mathrm{~A}-\mathrm{C} 2-\mathrm{H} 2 \mathrm{~B}$ & 109.0 \\
\hline $\mathrm{C} 5-\mathrm{N} 1-\mathrm{Cu} 1$ & $110.19(19)$ & $\mathrm{C} 3-\mathrm{C} 2-\mathrm{H} 2 \mathrm{~A}$ & 111.0 \\
\hline $\mathrm{C} 5-\mathrm{N} 1-\mathrm{C} 2$ & $105.7(2)$ & $\mathrm{C} 3-\mathrm{C} 2-\mathrm{H} 2 \mathrm{~B}$ & 111.0 \\
\hline $\mathrm{C} 6-\mathrm{N} 1-\mathrm{Cu} 1$ & $107.06(19)$ & $\mathrm{N} 23-\mathrm{C} 24-\mathrm{H} 24 \mathrm{~A}$ & 109.5 \\
\hline $\mathrm{C} 6-\mathrm{N} 1-\mathrm{C} 5$ & $109.8(2)$ & $\mathrm{N} 23-\mathrm{C} 24-\mathrm{H} 24 \mathrm{~B}$ & 109.5 \\
\hline $\mathrm{C} 6-\mathrm{N} 1-\mathrm{C} 2$ & $113.2(3)$ & $\mathrm{N} 23-\mathrm{C} 24-\mathrm{H} 24 \mathrm{C}$ & 109.5 \\
\hline $\mathrm{C} 2-\mathrm{N} 1-\mathrm{Cu} 1$ & $110.9(2)$ & $\mathrm{H} 24 \mathrm{~A}-\mathrm{C} 24-\mathrm{H} 24 \mathrm{~B}$ & 109.5 \\
\hline $\mathrm{C} 32-\mathrm{N} 31-\mathrm{Cu} 1$ & $134.3(2)$ & $\mathrm{H} 24 \mathrm{~A}-\mathrm{C} 24-\mathrm{H} 24 \mathrm{C}$ & 109.5 \\
\hline $\mathrm{C} 32-\mathrm{N} 31-\mathrm{C} 36$ & $106.1(3)$ & $\mathrm{H} 24 \mathrm{~B}-\mathrm{C} 24-\mathrm{H} 24 \mathrm{C}$ & 109.5 \\
\hline $\mathrm{C} 36-\mathrm{N} 31-\mathrm{Cu} 1$ & $119.2(2)$ & $\mathrm{N} 11-\mathrm{C} 16-\mathrm{H} 16$ & 124.8 \\
\hline $\mathrm{C} 22-\mathrm{N} 23-\mathrm{C} 25$ & $107.1(3)$ & $\mathrm{C} 15-\mathrm{C} 16-\mathrm{N} 11$ & $110.4(4)$ \\
\hline $\mathrm{C} 22-\mathrm{N} 23-\mathrm{C} 24$ & $125.8(3)$ & $\mathrm{C} 15-\mathrm{C} 16-\mathrm{H} 16$ & 124.8 \\
\hline $\mathrm{C} 25-\mathrm{N} 23-\mathrm{C} 24$ & $127.1(3)$ & $\mathrm{N} 4-\mathrm{C} 3-\mathrm{C} 2$ & $106.9(3)$ \\
\hline $\mathrm{C} 12-\mathrm{N} 11-\mathrm{Cu} 1$ & $111.1(2)$ & $\mathrm{N} 4-\mathrm{C} 3-\mathrm{H} 3 \mathrm{~A}$ & 110.3 \\
\hline
\end{tabular}




\begin{tabular}{|c|c|c|c|}
\hline $\mathrm{C} 12-\mathrm{N} 11-\mathrm{C} 16$ & $105.5(3)$ & $\mathrm{N} 4-\mathrm{C} 3-\mathrm{H} 3 \mathrm{~B}$ & 110.3 \\
\hline $\mathrm{C} 16-\mathrm{N} 11-\mathrm{Cu} 1$ & $141.8(3)$ & $\mathrm{C} 2-\mathrm{C} 3-\mathrm{H} 3 \mathrm{~A}$ & 110.3 \\
\hline $\mathrm{C} 32-\mathrm{N} 33-\mathrm{C} 35$ & $107.5(3)$ & $\mathrm{C} 2-\mathrm{C} 3-\mathrm{H} 3 \mathrm{~B}$ & 110.3 \\
\hline $\mathrm{C} 32-\mathrm{N} 33-\mathrm{C} 34$ & $128.3(3)$ & $\mathrm{H} 3 \mathrm{~A}-\mathrm{C} 3-\mathrm{H} 3 \mathrm{~B}$ & 108.6 \\
\hline $\mathrm{C} 35-\mathrm{N} 33-\mathrm{C} 34$ & $124.2(3)$ & $\mathrm{N} 13-\mathrm{C} 15-\mathrm{H} 15$ & 126.9 \\
\hline $\mathrm{C} 12-\mathrm{N} 13-\mathrm{C} 15$ & $106.8(3)$ & $\mathrm{C} 16-\mathrm{C} 15-\mathrm{N} 13$ & $106.2(3)$ \\
\hline $\mathrm{C} 12-\mathrm{N} 13-\mathrm{C} 14$ & $127.2(3)$ & $\mathrm{C} 16-\mathrm{C} 15-\mathrm{H} 15$ & 126.9 \\
\hline $\mathrm{C} 15-\mathrm{N} 13-\mathrm{C} 14$ & $126.0(3)$ & $\mathrm{N} 33-\mathrm{C} 34-\mathrm{H} 34 \mathrm{~A}$ & 109.5 \\
\hline $\mathrm{C} 5-\mathrm{N} 4-\mathrm{C} 7$ & $118.8(3)$ & N33-C34-H34B & 109.5 \\
\hline $\mathrm{C} 5-\mathrm{N} 4-\mathrm{C} 3$ & $103.6(3)$ & $\mathrm{N} 33-\mathrm{C} 34-\mathrm{H} 34 \mathrm{C}$ & 109.5 \\
\hline $\mathrm{C} 7-\mathrm{N} 4-\mathrm{C} 3$ & $116.4(3)$ & $\mathrm{H} 34 \mathrm{~A}-\mathrm{C} 34-\mathrm{H} 34 \mathrm{~B}$ & 109.5 \\
\hline $\mathrm{N} 11-\mathrm{C} 12-\mathrm{N} 13$ & $111.2(3)$ & $\mathrm{H} 34 \mathrm{~A}-\mathrm{C} 34-\mathrm{H} 34 \mathrm{C}$ & 109.5 \\
\hline $\mathrm{N} 11-\mathrm{C} 12-\mathrm{C} 5$ & $122.0(3)$ & $\mathrm{H} 34 \mathrm{~B}-\mathrm{C} 34-\mathrm{H} 34 \mathrm{C}$ & 109.5 \\
\hline $\mathrm{N} 13-\mathrm{C} 12-\mathrm{C} 5$ & $126.9(3)$ & $\mathrm{N} 13-\mathrm{C} 14-\mathrm{H} 14 \mathrm{~A}$ & 109.5 \\
\hline $\mathrm{N} 21-\mathrm{C} 26-\mathrm{H} 26$ & 125.7 & $\mathrm{~N} 13-\mathrm{C} 14-\mathrm{H} 14 \mathrm{~B}$ & 109.5 \\
\hline $\mathrm{C} 25-\mathrm{C} 26-\mathrm{N} 21$ & $108.5(3)$ & $\mathrm{N} 13-\mathrm{C} 14-\mathrm{H} 14 \mathrm{C}$ & 109.5 \\
\hline $\mathrm{C} 25-\mathrm{C} 26-\mathrm{H} 26$ & 125.7 & $\mathrm{H} 14 \mathrm{~A}-\mathrm{C} 14-\mathrm{H} 14 \mathrm{~B}$ & 109.5 \\
\hline $\mathrm{N} 21-\mathrm{C} 22-\mathrm{N} 23$ & $110.7(3)$ & $\mathrm{H} 14 \mathrm{~A}-\mathrm{C} 14-\mathrm{H} 14 \mathrm{C}$ & 109.5 \\
\hline $\mathrm{N} 21-\mathrm{C} 22-\mathrm{C} 6$ & $121.3(3)$ & $\mathrm{H} 14 \mathrm{~B}-\mathrm{C} 14-\mathrm{H} 14 \mathrm{C}$ & 109.5 \\
\hline $\mathrm{N} 23-\mathrm{C} 22-\mathrm{C} 6$ & $127.9(3)$ & & \\
\hline $\mathrm{Cu} 1-\mathrm{N} 21-\mathrm{C} 26-\mathrm{C} 25$ & $-169.6(3)$ & $\mathrm{C} 5-\mathrm{N} 4-\mathrm{C} 3-\mathrm{C} 2$ & $33.7(4)$ \\
\hline $\mathrm{Cu} 1-\mathrm{N} 21-\mathrm{C} 22-\mathrm{N} 23$ & $172.2(2)$ & $\mathrm{C} 32-\mathrm{N} 31-\mathrm{C} 36-\mathrm{C} 35$ & $-0.9(4)$ \\
\hline $\mathrm{Cu} 1-\mathrm{N} 21-\mathrm{C} 22-\mathrm{C} 6$ & $-3.5(4)$ & $\mathrm{C} 32-\mathrm{N} 33-\mathrm{C} 35-\mathrm{C} 36$ & $-0.1(4)$ \\
\hline $\mathrm{Cu} 1-\mathrm{N} 1-\mathrm{C} 5-\mathrm{N} 4$ & $-94.3(2)$ & $\mathrm{C} 6-\mathrm{N} 1-\mathrm{C} 5-\mathrm{N} 4$ & $148.0(3)$ \\
\hline $\mathrm{Cu} 1-\mathrm{N} 1-\mathrm{C} 5-\mathrm{C} 12$ & $31.5(3)$ & $\mathrm{C} 6-\mathrm{N} 1-\mathrm{C} 5-\mathrm{C} 12$ & $-86.2(3)$ \\
\hline $\mathrm{Cu} 1-\mathrm{N} 1-\mathrm{C} 6-\mathrm{C} 22$ & $30.9(3)$ & $\mathrm{C} 6-\mathrm{N} 1-\mathrm{C} 2-\mathrm{C} 3$ & $-124.7(3)$ \\
\hline $\mathrm{Cu} 1-\mathrm{N} 1-\mathrm{C} 2-\mathrm{C} 3$ & $114.9(3)$ & $\mathrm{C} 36-\mathrm{N} 31-\mathrm{C} 32-\mathrm{N} 33$ & $0.8(4)$ \\
\hline $\mathrm{Cu} 1-\mathrm{N} 31-\mathrm{C} 32-\mathrm{N} 33$ & $-171.5(2)$ & $\mathrm{C} 36-\mathrm{N} 31-\mathrm{C} 32-\mathrm{C} 7$ & $175.2(4)$ \\
\hline $\mathrm{Cu} 1-\mathrm{N} 31-\mathrm{C} 32-\mathrm{C} 7$ & $3.0(6)$ & $\mathrm{C} 25-\mathrm{N} 23-\mathrm{C} 22-\mathrm{N} 21$ & $0.4(4)$ \\
\hline $\mathrm{Cu} 1-\mathrm{N} 31-\mathrm{C} 36-\mathrm{C} 35$ & $172.8(3)$ & $\mathrm{C} 25-\mathrm{N} 23-\mathrm{C} 22-\mathrm{C} 6$ & $175.8(3)$ \\
\hline $\mathrm{Cu} 1-\mathrm{N} 11-\mathrm{C} 12-\mathrm{N} 13$ & $169.3(2)$ & $\mathrm{C} 35-\mathrm{N} 33-\mathrm{C} 32-\mathrm{N} 31$ & $-0.4(4)$ \\
\hline $\mathrm{Cu} 1-\mathrm{N} 11-\mathrm{C} 12-\mathrm{C} 5$ & $-11.4(4)$ & $\mathrm{C} 35-\mathrm{N} 33-\mathrm{C} 32-\mathrm{C} 7$ & $-175.5(3)$ \\
\hline $\mathrm{Cu} 1-\mathrm{N} 11-\mathrm{C} 16-\mathrm{C} 15$ & $-163.7(3)$ & $\mathrm{C} 7-\mathrm{N} 4-\mathrm{C} 5-\mathrm{N} 1$ & $94.3(3)$ \\
\hline $\mathrm{N} 21-\mathrm{C} 26-\mathrm{C} 25-\mathrm{N} 23$ & $0.4(4)$ & $\mathrm{C} 7-\mathrm{N} 4-\mathrm{C} 5-\mathrm{C} 12$ & $-26.5(4)$ \\
\hline $\mathrm{N} 21-\mathrm{C} 22-\mathrm{C} 6-\mathrm{N} 1$ & $-20.1(4)$ & $\mathrm{C} 7-\mathrm{N} 4-\mathrm{C} 3-\mathrm{C} 2$ & $-98.7(4)$ \\
\hline $\mathrm{N} 1-\mathrm{C} 2-\mathrm{C} 3-\mathrm{N} 4$ & $-17.7(4)$ & $\mathrm{C} 2-\mathrm{N} 1-\mathrm{C} 5-\mathrm{N} 4$ & $25.6(3)$ \\
\hline $\mathrm{N} 31-\mathrm{C} 32-\mathrm{C} 7-\mathrm{N} 4$ & $38.0(6)$ & $\mathrm{C} 2-\mathrm{N} 1-\mathrm{C} 5-\mathrm{C} 12$ & $151.4(3)$ \\
\hline $\mathrm{N} 31-\mathrm{C} 36-\mathrm{C} 35-\mathrm{N} 33$ & $0.6(4)$ & $\mathrm{C} 2-\mathrm{N} 1-\mathrm{C} 6-\mathrm{C} 22$ & $-91.6(3)$ \\
\hline $\mathrm{N} 23-\mathrm{C} 22-\mathrm{C} 6-\mathrm{N} 1$ & $165.0(3)$ & $\mathrm{C} 24-\mathrm{N} 23-\mathrm{C} 22-\mathrm{N} 21$ & $-179.5(3)$ \\
\hline $\mathrm{N} 11-\mathrm{C} 12-\mathrm{C} 5-\mathrm{N} 1$ & $-13.4(4)$ & $\mathrm{C} 24-\mathrm{N} 23-\mathrm{C} 22-\mathrm{C} 6$ & $-4.2(5)$ \\
\hline $\mathrm{N} 11-\mathrm{C} 12-\mathrm{C} 5-\mathrm{N} 4$ & $106.4(4)$ & $\mathrm{C} 24-\mathrm{N} 23-\mathrm{C} 25-\mathrm{C} 26$ & $179.4(3)$ \\
\hline $\mathrm{N} 11-\mathrm{C} 16-\mathrm{C} 15-\mathrm{N} 13$ & $0.8(4)$ & $\mathrm{C} 16-\mathrm{N} 11-\mathrm{C} 12-\mathrm{N} 13$ & $0.5(4)$ \\
\hline $\mathrm{N} 33-\mathrm{C} 32-\mathrm{C} 7-\mathrm{N} 4$ & $-148.0(3)$ & $\mathrm{C} 16-\mathrm{N} 11-\mathrm{C} 12-\mathrm{C} 5$ & $179.8(3)$ \\
\hline $\mathrm{N} 13-\mathrm{C} 12-\mathrm{C} 5-\mathrm{N} 1$ & $165.8(3)$ & $\mathrm{C} 3-\mathrm{N} 4-\mathrm{C} 5-\mathrm{N} 1$ & $-36.7(3)$ \\
\hline $\mathrm{N} 13-\mathrm{C} 12-\mathrm{C} 5-\mathrm{N} 4$ & $-74.4(4)$ & $\mathrm{C} 3-\mathrm{N} 4-\mathrm{C} 5-\mathrm{C} 12$ & $-157.5(3)$ \\
\hline $\mathrm{C} 12-\mathrm{N} 11-\mathrm{C} 16-\mathrm{C} 15$ & $-0.8(4)$ & $\mathrm{C} 3-\mathrm{N} 4-\mathrm{C} 7-\mathrm{C} 32$ & $54.2(5)$ \\
\hline $\mathrm{C} 12-\mathrm{N} 13-\mathrm{C} 15-\mathrm{C} 16$ & $-0.5(4)$ & $\mathrm{C} 15-\mathrm{N} 13-\mathrm{C} 12-\mathrm{N} 11$ & $0.0(4)$ \\
\hline
\end{tabular}




$\begin{array}{llll}\mathrm{C} 26-\mathrm{N} 21-\mathrm{C} 22-\mathrm{N} 23 & -0.1(4) & \mathrm{C} 15-\mathrm{N} 13-\mathrm{C} 12-\mathrm{C} 5 & -179.3(3) \\ \mathrm{C} 26-\mathrm{N} 21-\mathrm{C} 22-\mathrm{C} 6 & -175.9(3) & \mathrm{C} 34-\mathrm{N} 33-\mathrm{C} 32-\mathrm{N} 31 & -178.2(4) \\ \mathrm{C} 22-\mathrm{N} 21-\mathrm{C} 26-\mathrm{C} 25 & -0.2(4) & \mathrm{C} 34-\mathrm{N} 33-\mathrm{C} 32-\mathrm{C} 7 & 6.7(6) \\ \mathrm{C} 22-\mathrm{N} 23-\mathrm{C} 25-\mathrm{C} 26 & -0.5(4) & \mathrm{C} 34-\mathrm{N} 33-\mathrm{C} 35-\mathrm{C} 36 & 177.8(4) \\ \mathrm{C} 5-\mathrm{N} 1-\mathrm{C} 6-\mathrm{C} 22 & 150.5(3) & \mathrm{C} 14-\mathrm{N} 13-\mathrm{C} 12-\mathrm{N} 11 & 178.4(4) \\ \mathrm{C} 5-\mathrm{N} 1-\mathrm{C} 2-\mathrm{C} 3 & -4.5(4) & \mathrm{C} 14-\mathrm{N} 13-\mathrm{C} 12-\mathrm{C} 5 & -0.9(6) \\ \mathrm{C} 5-\mathrm{N} 4-\mathrm{C} 7-\mathrm{C} 32 & -70.9(5) & \mathrm{C} 14-\mathrm{N} 13-\mathrm{C} 15-\mathrm{C} 16 & -178.9(4)\end{array}$

